\title{
ON THE CALCULATION OF SECULAR PERTURBATIONS IN THE CASE OF CLOSE COMMENSURABILITY
}

\author{
N. I. LOBKOVA and M. S. PETROVSKAYA \\ Institute of Theoretical Astronomy, Leningrad, U.S.S.R.
}

\begin{abstract}
In a previous paper the authors derived expansions of the derivatives of the disturbing function for the general case including the orbits close to intersection. The present paper deals especially with the case of close commensurability of the mean motions. A new variable $v$ is introduced characterizing the deviation of the mean anomalies from the exact commensurability, and is considered further as an unknown quantity. In the equations of motion the short-period terms are eliminated. The form of expansions of the right-hand sides is chosen basing on the same principles as in the general case. The factors are separated, corresponding to the poles in the case of circular intersecting orbits. For rapidity of calculation the summation in powers of the major semi-axes ratio is made the inner one.
\end{abstract}

\section{Expansion with Respect to the Negative Powers of the Mutual Distances}

For studying the case of close commensurability in the planetary motion von Zeipel's method and its various modifications are generally used. The canonical transformations performed in the methods entail the Hamiltonian expansions in trigonometric series, the elimination of particular arguments and finding the mean and extreme values of the Hamiltonian. All these operations are usually carried out numerically (Giacaglia, 1968; Hori and Giacaglia, 1968; Giacaglia and Nacozy, 1969), since the analytical treatment is complicated by the problem of convergence of the series. The well-known expansions of the disturbing function are valid, provided the ratio of the major axes and other parameters are sufficiently small (Kozai, 1962, 1968). In Petrovskaya (1972) an analytical expansion of the perturbation function is given for the general case, including the intersecting orbits. When resonance problems are considered the possibility offers to improve the convergence of the series. One can take advantage of the fact that the mean anomalies, $M_{1}$ and $M_{2}$, of the planets are fairly commensurable and so, the distance $\Delta$ between the bodies is approximately a periodic function of the time. While the convergence of the two-argument expansion representing $\Delta^{-\gamma}\left(M_{1}, M_{2}\right)$ depends on the minimum distance between the planetary orbits, the validity of the single-argument expansion of $\Delta^{-\gamma}$ in the case of exact commensurability is dependent on the minimum actual distance between the planets.

Considering the case of close commensurability we put

$$
M_{j}=M_{j}^{0}+\int_{t_{0}}^{t} N_{j} \mathrm{~d} t, \quad j=1,2,
$$

$N_{j}$ being the mean motions of the planets, and

$$
N_{2}-(p / q) N_{1}=v \text {, }
$$

where $p$ and $q$ are integers, and $v$ is a small quantity. 
Introduce, instead of $M_{1}$ and $M_{2}$, new variables $z$ and $y$ as follows:

$$
z=\frac{1}{q} \int_{t_{0}}^{t} N_{1} \mathrm{~d} t, \quad y=\int_{t_{0}}^{t} v \mathrm{~d} t .
$$

Hence $M_{1}$ and $M_{2}$ are expressed as

$$
M_{1}=q z+M_{1}^{0}, \quad M_{2}=p z+M_{2}^{0}+y .
$$

If $N_{1}$ and $N_{2}$ are strictly commensurable, that is $v=0$, we have $y=0$. Thus, $y$ is a slowly changing variable.

In the Lagrange's equations the disturbing function after substituting (2) becomes a function of $y$ and $z$, the equations for those being

$$
\begin{aligned}
\mathrm{d} y / \mathrm{d} t & =v, \\
\mathrm{~d} z / \mathrm{d} t & =N_{1} / q .
\end{aligned}
$$

In the restricted three-body problem one of the two $N_{j}$ is a constant. Let it be $N_{2}$. Then, after finding $y$ from Equation (3a), $M_{1}$ will be

$$
M_{1}=(q / p)\left(M_{2}-M_{2}^{0}-y\right)+M_{1}^{0} .
$$

We put $a$ : the major semi-axis, $e$ : the eccentricity, $i$ : the inclination, $\Omega$ : the longitude of the node, $\omega$ : the argument of the perihelion, $M^{0}:$ the mean anomaly at the epoch.

The mutual distance of the planets $\Delta\left(a, e, i, \Omega, \omega, M^{0}, z, y\right)$, where $a=a_{1}, a_{2}, \ldots$, in the case of rigorous commensurability for the undisturbed motion, is

$$
\Delta_{0}=\Delta\left(a^{0}, e^{0}, i^{0}, \Omega^{0}, \omega^{0}, M\left(t_{0}\right), z, 0\right),
$$

being the function of a single variable $z$ and of 12 constants.

Consider a Taylor's expansion,

$$
\Delta^{-1}=\Delta_{0}^{-1} \sum_{\kappa=0}^{\infty} \frac{\left(\frac{1}{2}\right)_{\kappa}}{(1)_{\kappa}}\left(1-\frac{\Delta^{2}}{\Delta_{0}^{2}}\right)^{\kappa}
$$

It is a series in powers of the deviation of $\Delta^{2}$ from $\Delta_{0}^{2}$, which is of order of $\sigma=\max$ $\{\mu,|v|\}$, where $\mu$ is the disturbing mass, and $v$ is given by (1). If, for example, $|v| \leqslant \mu^{1 / 2}$, then the terms of series (4) are of the order $\mu^{\kappa / 2}$. When $|v|>\mu^{1 / 2}$ there is no need to treat the problem as one of resonance, since in this case the perturbations may be derived by the usual procedure of successive approximations and the solution of the differential equations would be developed in powers of $\mu^{1 / 2}$.

Thus, we assume that $|v| \leqslant \mu^{1 / 2}$. Then, if the perturbations up to the second order with respect to $\mu$ have to be calculated, one should substitute into Lagrange's equations instead of $\Delta^{-1}$ the sum of terms of expansion (4) for $\kappa=1,2,3,4$. The zero term disappears after differentiation with respect to the elements. Hence, if one starts from (4) the problem of developing the principal part of the disturbing function is reduced to the expansion of several positive powers of the two-argument function $\Delta^{2}(z, y)$, 
and several negative powers of the single-argument function $\Delta_{0}^{2}(z)$, all the Keplerian elements in the latter being fixed.

One has to take notice of the following characteristics of expansion (4). It involves no negative powers of the two-argument distrubed function $\Delta(z, y)$ while the positive powers of $\Delta^{2}(z, y)$ are rapidly convergent series which can be easily differentiated with respect to Keplerian elements. The functions $\Delta_{0}^{-2 \kappa^{-1}}(z)$ are single-argument series which are not differentiated into Langrange's equations, their coefficients being evaluated once with any precision. It is convenient to use expansion (4) when the equations are solved with the short-period terms being eliminated since all the operations performed by numerical integration or by finding out the stationary solutions etc. are applied to the positive powers of $\Delta^{2}$, which depends analytically on the elements.

We confine ourselves to the evaluation of the perturbations up to the second order. As in (4) the same powers of $\Delta^{2} / \Delta_{0}^{2}$ appear several times, the following form of $\Delta^{-1}$ is preferable:

$$
\begin{aligned}
\Delta^{-1} & =\sum_{s=0}^{4} \lambda_{s} \frac{\Delta^{2 s}}{\Delta_{0}^{2 s+1}} \\
\lambda_{s} & =\frac{(-1)^{s}}{(1)_{s}}\left(\frac{1}{2}\right)_{s} \sum_{q=0}^{4-s} \frac{\left(\frac{1}{2}+s\right)_{q}}{(1)_{q}} .
\end{aligned}
$$

\section{Expansion of $\Delta^{2 s}$}

Consider

$$
\Delta^{2 s}=\left(\tau_{1}^{2}+\tau_{2}^{2}-2 \tau_{1} \tau_{2} \cos H\right)^{s},
$$

where $\tau_{1}, \tau_{2}$ and $H$ are the distances of Sun-asteroid, Sun-Jupiter and the elongation of the asteroid from Jupiter.

As it is known (Sack, 1964), the following relations hold:

$$
\begin{aligned}
& \Delta^{2 s}=\sum_{\kappa=0}^{s} R_{2 s, \kappa}\left(\tau_{1}, \tau_{2}\right) P_{\kappa}(\cos H) \\
& R_{2 s, \kappa}\left(\tau_{1}, \tau_{2}\right)=\tau_{2}^{2 s} \frac{(-s)_{\kappa}}{\left(\frac{1}{2}\right)_{\kappa}}\left(\frac{\tau_{1}}{\tau_{2}}\right)^{\kappa} F\left(\kappa-s,-\frac{1}{2}-s, \kappa+\frac{3}{2} ; \frac{\tau_{1}^{2}}{\tau_{2}^{2}}\right),
\end{aligned}
$$

or

$$
\Delta^{2 s}=\sum_{\kappa=0}^{s} \frac{(-s)_{\kappa}}{\left(\frac{1}{2}\right)_{\kappa}} P_{\kappa}(\cos H) \sum_{n=0}^{s-\kappa} \frac{(\kappa-s)_{n}\left(-\frac{1}{2}-s\right)_{n}}{\left(\kappa+\frac{3}{2}\right)_{n}(1)_{n}} \tau_{1}^{2 n+\kappa} \tau_{2}^{2 s-\kappa-2 n}
$$

The Legendre's polynominals $P_{\kappa}(\cos H)$ can be presented by the formula (Brumberg, 1971),

$$
\begin{aligned}
P_{\kappa}(\cos H)= & \sum_{l=0}^{\kappa} \sum_{l_{1}=0}^{\kappa} \sum_{j=0}^{\kappa}\left(2-\delta_{j, 0}\right) \frac{(1)_{\kappa-j}}{(1)_{\kappa+j}} F_{\kappa j l}\left(i_{1}\right) \times \\
& \times F_{\kappa j l_{1}}\left(i_{2}\right) \cos \left[(\kappa-2 l) u_{1}-\left(\kappa-2 l_{1}\right) u_{2}+j\left(\Omega_{1}-\Omega_{2}\right)\right],
\end{aligned}
$$


where $u_{1}$ and $u_{2}$ are the arguments of latitude of the planets and

$$
\begin{aligned}
& F_{\kappa j l}(i)=\lambda_{\kappa j l}\left(\sin \left(\frac{1}{2} i\right)\right)^{|\kappa-j-2 l|}\left(\cos \left(\frac{1}{2} i\right)\right)^{|\kappa+j-2 l|} \times \\
& \quad \times F\left(-m, 2 \kappa-m+1,1+|\kappa-j-2 l| ; \sin ^{2}\left(\frac{1}{2} i\right) .\right. \\
& m=\kappa-\frac{1}{2}(|\kappa-j-2 l|+|\kappa+j-2 l|),
\end{aligned}
$$

$\lambda$ are some numerical coefficients, $\delta_{0,0}=1, \delta_{j, 0}=0$ if $j \neq 0, F$ is Gauss' hypergeometric function.

After substituting (7) into (6) $\Delta^{2 s}$ can be readily presented as a series in the multiples of $M_{1}$ and $M_{2}$ :

$$
\Delta^{2 s}=\sum_{q_{1}} \sum_{q_{2}} \sum_{q_{3}, q_{4}, q_{5}} A_{\{q\}} \cos \left[q_{1} M_{1}+q_{2} M_{2}+q_{3} \omega_{1}+q_{4} \omega_{2}+q_{5}\left(\Omega_{1}-\Omega_{2}\right)\right] .
$$

The absolute values of the integers $q_{3}, q_{4}$ and $q_{5}$ do not exceed 4. $A_{\{q\}}$ are the functions of the remaining elements $a, e, i$ :

$$
A_{\{q\}}=C\left(a_{1}, a_{2}\right) X\left(e_{1}\right) X\left(e_{2}\right) F\left(i_{1}\right) F\left(i_{2}\right),
$$

$C$ and $F$ being the polynominals of the order $2 s$ and $X$ being Hansen's coefficients. The limits in the sums with respect to $q_{1}$ and $q_{2}$ depend on the rapidity of diminishing Hansen's coefficients, in other words, on the values of eccentricities.

Expression (9) may be presented as a series with respect to all the variables involved, so that it would be a polynomial in $a_{1}, a_{2}, \sin \left(\frac{1}{2} i_{1}\right)$ and $\sin \left(\frac{1}{2} i_{2}\right)$, a power series in $e_{1}$ and $e_{2}$, a trigonometric one with respect to $M_{1}$ and $M_{2}$ and a trigonometric polynomial in $\omega_{1}, \omega_{2}$, and $\Omega_{1}-\Omega_{2}$. This series will be obtained readily as soon as any of wellknown expansions of Hansen's coefficients in powers of eccentricities is used. In such a form function (9) and, therefore, (5) can be easily differentiated with respect to the Keplerian elements.

Substituting (2) into (9) it follows:

$$
\Delta^{2 s}=\sum_{n=-n_{2}}^{n_{2}} B_{n}^{(2 s)} e^{i n z}, \quad n_{1}>0, \quad n_{2}>0,
$$

$B_{n}^{(2 s)}$ being the function of Keplerian elements and $y$. The number of terms taken into consideration, as was mentioned above, depends on the values of the eccentricities.

\section{Expansion of $\Delta_{0}^{-\gamma}, \gamma=1,3,5, \ldots$}

Now we would like to express $\Delta_{0}^{-\gamma}$ as a Fourier series in $z$ with numerical coefficients. The first step is to develop $\Delta_{0}^{-\gamma}$ in multiples of $M_{1}$ and $M_{2}$ by any device of expansion. Taking into account that in $\Delta_{0} M_{j}$ are given by (2) under condition $y=0$ one is to construct the expansion

$$
\Delta_{0}^{-\gamma}=\sum_{n=-\infty}^{\infty} C_{n}^{(\gamma)} e^{i n z}, \quad \gamma=1,3, \ldots
$$

In order to calculate the coefficients $C_{n}^{(\gamma)}$ an intermediate expansion of $\Delta^{-\gamma}$ may be 
applied which was derived by Petrovskaya (1972). From this expansion $C_{n}^{(\gamma)}$ are defined as

$$
\begin{aligned}
C_{n}^{(\gamma)} & =\sum_{k=0}^{\infty} \sum_{m=0}^{\infty} \frac{(D)_{\kappa}}{(1)_{\kappa}} \Phi_{\kappa}^{(\gamma)}(\beta) I_{n \kappa m}^{(\gamma)}, \\
I_{n \kappa m}^{(\gamma)} & =\frac{1}{2 \pi} \int_{0}^{2 \pi} f_{\kappa, m}^{(\gamma)}(z) e^{-i n z} \mathrm{~d} z \\
f_{\kappa, m}^{(\gamma)}(z) & =\left(\tau_{1}^{0}+\tau_{2}^{0}\right)^{-\gamma} x^{\kappa} \cos m H^{0}, \\
D & =\frac{\partial}{\partial \ln \beta}, \quad \beta=\frac{a_{1}^{0} a_{2}^{0}\left(1-e_{1}^{0}\right)\left(1-e_{2}^{0}\right)}{\left[a_{1}^{0}\left(1+e_{1}^{0}\right)+a_{2}^{0}\left(1+e_{2}^{0}\right)\right]^{2}}, \\
\Phi_{m}^{(\gamma)}(\beta) & =\left(2-\delta_{m, 0}\right) \frac{(\gamma / 2)_{m}}{(1)_{m}} \beta^{m} F\left(m+\frac{1}{2} \gamma, m+\frac{1}{2} \gamma, 2 m+1 ; 4 \beta\right), \\
x & =1-\beta\left(\tau_{1}^{0}+\tau_{2}^{0}\right)^{2} / \tau_{1}^{0} \tau_{2}^{0} .
\end{aligned}
$$

As to the integrals $I_{n \kappa m}^{(\gamma)}$ they may be evaluated either by mechanical quadratures methods or by developing them in an analytical way with respect to Keplerian elements. In order to obtain an analytical expansion the functions $f_{\kappa, m}^{(\gamma)}(z)$ have to be presented as a series in multiples of $M_{1}$ and $M_{2}$ and then of $z$, taking into consideration that

$$
M_{1}=q z+M_{1}\left(t_{0}\right), \quad M_{2}=p z+M_{2}\left(t_{0}\right) .
$$

Finally, one can develop with respect to $z$ each function of $\left(\tau^{0}+\tau_{2}^{0}\right)^{-\gamma}, x$ and $\cos H^{0}$ in (14) and the series for (14) follows from the multiplications.

To calculate the functions $(D)_{\kappa} \Phi_{m}^{(\gamma)}(\beta)$ in (12) recurrent formulas were obtained earlier. These functions can be also evaluated without application of recurrent relations which sometimes may result in less accurate calculations. The following formula is free from that deficiency:

$$
\begin{aligned}
& \frac{(D)_{\kappa}}{(1)_{\kappa}} \Phi_{m}^{(\gamma)}(\beta)=(1-4 \beta)^{1 / 2-\gamma / 2-\kappa}(m)_{\kappa} \times \sum_{i=0}^{\kappa} A_{m i \kappa} \Phi_{m i}^{(\gamma)}, \\
& \Phi_{m i}^{(\gamma)}=\left(2-\delta_{m, 0}\right) \frac{(\gamma / 2)_{m}}{(1)_{m}} \beta^{m} F\left(m+1-\frac{1}{2} \gamma, m+\frac{1}{2}, 2 m+1+i ; 4 \beta\right), \\
& A_{\text {miк }}=\frac{(4 \beta)^{i}\left(m+\frac{1}{2} \gamma\right)_{i}\left(m+\frac{1}{2}\right)_{i}(1-4 \beta)^{\kappa-i}}{(1)_{i}(m)_{i}(2 m+1)_{i}(1)_{\kappa-i}} .
\end{aligned}
$$

Formulas (11)-(15) provide the expansion of $\Delta_{0}^{-\gamma}$.

The coefficients of the series (11) might be calculated by evaluating the integrals

$$
C_{n}^{(\gamma)}=\frac{1}{2 \pi} \int_{0}^{2 \pi} \Delta_{0}^{-\gamma} e^{-i n z} \mathrm{~d} z,
$$

with the aid of the quadrature formulas. Though this way may lead to difficulties when 
the minimum distance between the orbits is small $(\Delta \rightarrow 0)$. In our procedure we suggest to use the harmonic analysis method only for calculating the functions (13), after the singularity $(1-4 \beta)^{1 / 2-\gamma / 2-\kappa}$ at $a_{1}^{0} \rightarrow a_{2}^{0}, e_{1}^{0} \rightarrow 0, e_{2}^{0} \rightarrow 0$ is removed from (11) by the formula (15). The absolute value of the integrand $f_{\kappa, m}^{(\gamma)}$ in (13) is less than 1 .

\section{Averaged Equations of Motion}

For simplicity we consider the restricted three-body problem. The formulas (5), (10) and (11) provide the expansion of $\Delta^{-1}$ with respect to $z$. The constant term in $\Delta^{-1}$ is

$$
\left[\Delta^{-1}\right]=\sum_{s=0}^{4} \lambda_{s} \sum_{n=-n_{1}}^{n_{2}} B_{n}^{(2 s)} C_{-n}^{(2 s+1)},
$$

where $C_{-n}^{(2 s+1)}$ are numerical coefficients, while $B_{n}^{(2 s)}$ are functions of the disturbed Keplerian elements. The limits $n_{1}$ and $n_{2}$ are defined by the number of terms retained in $\Delta^{2 s}$.

After substituting (16) into Lagrange's equations the secular and long-period perturbations will be determined by the set

$$
\mathrm{d} \varepsilon / \mathrm{d} t=\mu f(\varepsilon, y), \quad \mathrm{d} y / \mathrm{d} t=N_{2}-(p / q) N_{1}=v,
$$

$\varepsilon$ being any Keplerian element, $N_{2}=$ const, $N_{1}$ being a function of $a_{1}, f(\varepsilon, y)$ being derivatives of the quantity (16).

After solving (17), $M_{1}$ is provided by the relations

$$
M_{1}=(q / p)\left(M_{2}-M_{2}^{0}-y\right)+M_{1}^{0}, \quad M_{2}=N_{2}\left(t-t_{0}\right) .
$$

We denote

$$
v=v_{0}\left(1+v_{1}\right)
$$

where $v_{0}$ is the value of $v$ for $\mu=0$. It is easily seen that, if $v_{0}$ is less than $\mu^{1 / 2}$, by putting $\tau=v_{0}\left(t-t_{0}\right)$ and $y=\tau+\zeta$ one obtains equations

$$
\mathrm{d} \varepsilon / \mathrm{d} \tau=\lambda f(\varepsilon, \tau+\zeta), \quad \mathrm{d} \zeta / \mathrm{d} \tau=v_{1},
$$

where $\lambda=O\left(\mu^{1 / 2}\right)$.

After averaging with respect to $\tau$ the equations provide the purely secular perturbations with respect to time, which are power series in $\lambda$. The periodic terms in the solution can be found by the usual procedure of von Zeipel.

In the case which is of particular interest for us the order of $v$ is more than $\mu^{1 / 2}$ and we return to set (17). If it is solved by numerical integration then at any step of approximation the coefficients $C_{-n}^{(2 s+1)}$ in (16) corresponding to the expansion (11) remain unchanged. They may be evaluated once with any precision. The coefficients $B_{n}^{(2 s)}$ which appear in (16) from (10) are literal series with respect to all the Keplerian elements, including the major semi-axes.

Equations (17) may be applied for finding out periodic solutions and those which are close to them. 
This consideration remains valid in the case of intersecting resonant oribts of asteroids and in the Neptune-Pluto case.

\section{References}

Brumberg, V. A., Evdokimova, L. S., and Kochina, N. G.: 1971, Celes. Mech. 3, 197.

Giacaglia, G. E. O.: 1968, Smithsonian Astrophys. Obs., Special Report No. 278.

Giacaglia, G. E. O. and Nacozy, P. E.: 1969, in G. E. O. Giacaglia (ed.), Periodic Orbits, Stability and Resonances, D. Reidel, Dordrecht, The Netherlands, p. 96.

Hori, G. and Giacaglia, G. E. O.: 1968, Research in Celestial Mechanics and Differential Equations (University of Paulo) 1, 4.

Kozai, Y.: 1962, Astron. J. 67, 591.

Kozai, Y.: 1968, in G. E. O. Giacaglia (ed.), Periodic Orbits, Stability and Resonances, D. Reidel, Dordrecht, The Netherlands, p. 451.

Petrovskaya, M. S.: 1972, Celes. Mech. 6, 328.

Sack, R. A.: 1964, J. Math. Phys. 5, 245. 